\title{
A cold/ultracold neutron detector using fine-grained nuclear emulsion with spatial resolution less than $100 \mathrm{~nm}$
}

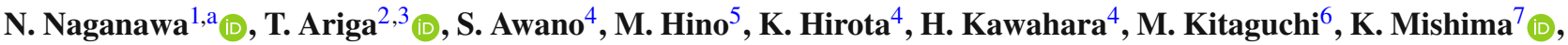 \\ H. M. Shimizu ${ }^{4}$, S. Tada ${ }^{4}$, S. Tasaki ${ }^{8}$, A. Umemoto ${ }^{4}$ \\ ${ }^{1}$ Institute of Materials and Systems for Sustainability, Nagoya University, Chikusa, Nagoya 464-8602, Japan \\ ${ }^{2}$ Faculty of Arts and Science, Kyushu University, Fukuoka 819-0395, Japan \\ ${ }^{3}$ Laboratory for High Energy Physics, University of Bern, 3012 Bern, Switzerland \\ ${ }^{4}$ Department of Physics, Nagoya University, Chikusa, Nagoya 464-8602, Japan \\ ${ }_{6}^{5}$ Institute for Integrated Radiation and Nuclear Science, Kyoto University, Kumatori, Osaka 590-0494, Japan \\ ${ }^{6}$ Center for Experimental Studies, KMI, Nagoya University, Chikusa, Nagoya 464-8602, Japan \\ ${ }^{7}$ High Energy Accelerator Research Organization, Tokai, Ibaraki 319-1106, Japan \\ ${ }^{8}$ Department of Nuclear Engineering, Kyoto University, Kyoto 615-8540, Japan
}

Received: 23 April 2018 / Accepted: 30 October 2018 / Published online: 21 November 2018

(C) The Author(s) 2018

\begin{abstract}
A new type of cold/ultracold neutron detector that can realize a spatial resolution of less than $100 \mathrm{~nm}$ was developed using nuclear emulsion. The detector consists of a fine-grained nuclear emulsion coating and a 50-nm thick ${ }^{10} \mathrm{~B}_{4} \mathrm{C}$ layer for the neutron conversion. The detector was exposed to cold and ultracold neutrons (UCNs) at the JPARC. Detection efficiencies were measured as $(0.163 \pm$ 0.015 (stat) \pm 0.013 (sys))\% and $(10.3 \pm 1.3$ (stat) \pm 1.1 (sys)) $\%$ for cold and ultracold neutrons consistently with the ${ }^{10} \mathrm{~B}$ content in the converter. Positions of individual neutrons can be determined by observing secondary particle tracks recorded in the nuclear emulsion. The spatial resolution of incident neutrons were found to be in the range of 11-99 $\mathrm{nm}$ in the angle region of $\tan \theta \leq 1.9$, where $\theta$ is the angle between a recorded track and the normal direction of the converter layer. The achieved spatial resolution corresponds to the improvement of one or two orders of magnitude compared with conventional techniques and it is comparable with the wavelength of UCNs.
\end{abstract}

\section{Introduction}

Nuclear emulsion is a type of photographic film used as a tracking device in particle physics, which features high spatial resolution. For a directional dark matter search, finegrained nuclear emulsions were produced with silver halide crystals of 35-nm diameter [1-3]. We succeeded in devel-

\footnotetext{
a e-mail: naganawa@flab.phys.nagoya-u.ac.jp
}

oping a neutron detector which realizes a spatial resolution of less than $100 \mathrm{~nm}$ by using the emulsion and a thin layer which includes ${ }^{10} \mathrm{~B}$, as a neutron converter. The improved resolution, which is better than that of conventional detectors $(1-2 \mu \mathrm{m})[4,5]$ by one or two orders of magnitude, allows for numerous possibilities and new applications. For example, this detector is suitable for measurements of position distributions of quantized states under the influence of the earth's gravitational field [6-8], or in searches of unknown short range forces. The high spatial resolution is also quite useful in searches for electric charges associated with neutrons $[9,10]$. This detector facilitates measurements of matter waves of neutrons because their wavelength with regard to ultracold neutrons (UCNs) is $\sim 60 \mathrm{~nm}$.

\section{High spatial resolution nuclear emulsion detector}

The detector consists of a thin converter layer formed on a 0.4 -mm-thick silicon substrate, which is coated with $10-\mu \mathrm{m}$ thick fine-grained nuclear emulsion. The converter consists of ${ }^{10} \mathrm{~B}_{4} \mathrm{C}(50 \mathrm{~nm}), \mathrm{NiC}(60 \mathrm{~nm})$, and $\mathrm{C}(30 \mathrm{~nm})$ layers (Fig. 1). The converter was fabricated using an ion beam sputtering machine at KURRI [11] and the thicknesses of the layers were estimated based on deposition rates. ${ }^{10} \mathrm{~B}_{4} \mathrm{C}$ converts neutrons into two ions whereas the $\mathrm{NiC}$ layer stabilizes the ${ }^{10} \mathrm{~B}_{4} \mathrm{C}$ layer. The $\mathrm{C}$ layer is used for chemical protection and a smooth adhesion for the emulsion. In order to determine the amount of ${ }^{10} \mathrm{~B}$ in the ${ }^{10} \mathrm{~B}_{4} \mathrm{C}$ layer, neutron transmissions of two $\mathrm{Si}^{-10} \mathrm{~B}_{4} \mathrm{C}-\mathrm{NiC}-\mathrm{C}$ plates, which were fabricated together with the detector's, were measured with cold neu- 


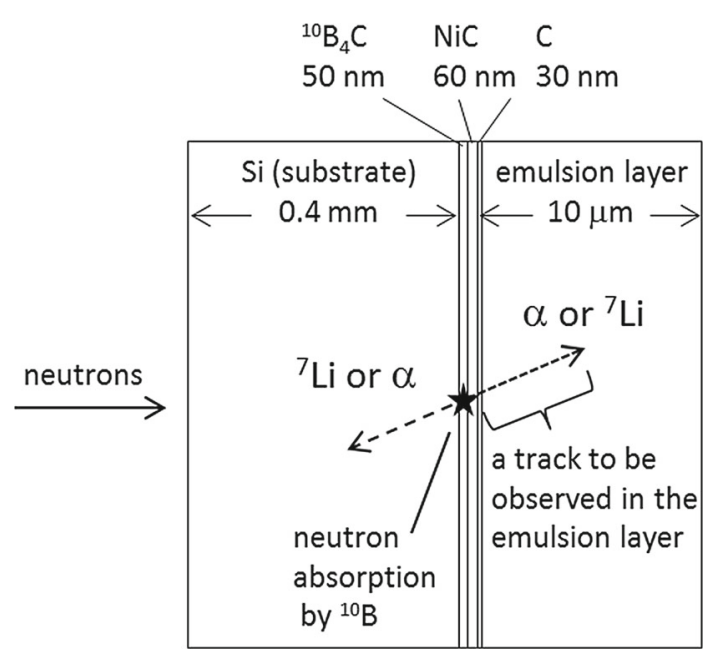

Fig. 1 A schematic view of the detector. When a neutron is absorbed in the ${ }^{10} \mathrm{~B}_{4} \mathrm{C}$ layer, two ions, an $\alpha$-particle and a ${ }^{7} \mathrm{Li}$ nucleus, are emitted. One of them is detected as a track which is several microns long in the emulsion layer

trons at BL05 of J-PARC MLF $[12,13]$. From the average of them, an absorption rate was evaluated at $(0.136 \pm 0.003$ (stat) \pm 0.038 (sys) $) \%$ for $1000 \mathrm{~m} / \mathrm{s}$ neutrons, which corresponds to a ${ }^{10} \mathrm{~B}$ content of $(1.61 \pm 0.03$ (stat) \pm 0.45 (sys) $\times 10^{17}$ nuclei $/ \mathrm{cm}^{2}$. The contributions to those systematic errors were from the standard deviation of cold neutron transmission of the two plates (28\%) and the uncertainty of angles of plates against the cold neutron beam during the transmission measurement $(1.9 \%)$ in relative errors. The latter was 1 mrad for the exposure angle of $52.4 \mathrm{mrad}$.

The fine-grained nuclear emulsion consists of $\mathrm{AgBr} \cdot \mathrm{I}$ crystals with a diameter of $35 \mathrm{~nm}$ and gelatin. When a charged particle penetrates the crystals, latent images are formed in them. The development (photographic processing) of these latent images as photographic films using chemical solutions, results in the generation of silver grains with a diameter of $\sim$ $100 \mathrm{~nm}$. During the development, the thickness of the emulsion layer shrinks to 0.6 times of the original. The induced tracks are detected using an optical microscope with an epiilluminat-ion system. This emulsion is optimized for heavily ionizing particles due to neutron absorption, but insensitive to electron and gamma-ray backgrounds. To detect slow neutrons, absorption by ${ }^{10} \mathrm{~B}$ was used:

$n+{ }^{10} \mathrm{~B} \rightarrow \alpha+{ }^{7} \mathrm{Li}+2.79 \mathrm{MeV}(6 \%)$

$n+{ }^{10} \mathrm{~B} \rightarrow \alpha+{ }^{7} \mathrm{Li}^{*}+\gamma(0.48 \mathrm{MeV})+2.31 \mathrm{MeV}(94 \%)$

where ${ }^{7} \mathrm{Li}^{*}$ is the first excitation state of ${ }^{7} \mathrm{Li} . \alpha$-particles and ${ }^{7} \mathrm{Li}$ nuclei from those reactions are detected in the nuclear emulsion. In the case of reaction (2), $\alpha$-particles and ${ }^{7} \mathrm{Li}$ nuclei form tracks with ranges of $(5.1 \pm 0.4) \mu \mathrm{m}$ and $(2.6 \pm$
0.4) $\mu \mathrm{m}$, respectively, in the fine-grained nuclear emulsion based on calculation by SRIM-2008.04. Their errors show stragglings of the ranges. After an absorption, an $\alpha$-particle and $\mathrm{a}^{7} \mathrm{Li}$ nucleus are emitted in the opposite directions. One of them enters the emulsion layer and is detected. By retracing the track to the converter layer, the position of the absorption point can be estimated using the distribution of grains along the track as will be discussed later.

There are three kinds of background. First one is $\alpha$ particles from natural radiations from thorium and uranium chains. Ranges of them are longer than $16 \mu \mathrm{m}$ in the emulsion layer from calculations based on SRIM-2008.04. Second one is protons recoiled by fast neutrons in the beamline. Third one is protons from ${ }^{14} \mathrm{~N}(\mathrm{n}, \mathrm{p})$ reactions caused by nitrogen originally contained in emulsion layer with its mass fraction of $(0.027 \pm 0.003) \mathrm{g} / \mathrm{g}[3]$. This value corresponds to ${ }^{14} \mathrm{~N}$ content of $(4.0 \pm 0.4) \times 10^{18}$ nuclei $/ \mathrm{cm}^{2}$ in $10-\mu \mathrm{m}$-thickness. Protons from the reactions have the range of $(7.0 \pm 0.5)$ $\mu \mathrm{m}$ based on calculation by SRIM-2008.04. The emulsion layer of $10-\mu \mathrm{m}$-thick can sufficiently facilitate rejection of background tracks. Firstly, tracks which are not attached to the sputtered layer are all recognized as backgrounds. By this, most of short background tracks of recoiled protons and those from ${ }^{14} \mathrm{~N}(\mathrm{n}, \mathrm{p})$ reaction are rejected. Secondly, tracks attached to the sputtered layer which are longer than the lengths of signal tracks, i.e. longer than $\alpha$-tracks from (1) or (2), are rejected. In such a manner, $\alpha$-tracks from isotopes outside of emulsion layer belonging to thorium or uranium chain or tracks of recoiled protons which are longer than signals attached to the sputtered layer are rejected. Thirdly, tracks of recoiled protons which are shorter than signals and attached to the sputtered layer by chance are rejected by recognizing their low density of silver grains due to whose ionization loss being less than the half of signal tracks. Also multiple coulomb scattering which is significantly larger than signal tracks can be used for the rejection. Fourthly, $\alpha$-tracks whose sources are inside of the emulsion layer are rejected by recognizing topologies of multiple tracks from the decay chain starting from the positions of single sources.

For coating the converter layer with emulsion gel, it was melted at $40^{\circ} \mathrm{C}$ and was taken by a micropipette, deposited on $\mathrm{Si}^{-}{ }^{10} \mathrm{~B}_{4} \mathrm{C}-\mathrm{NiC}-\mathrm{C}$ plates of dimensions $2 \mathrm{~cm} \times 2 \mathrm{~cm}$, and spread using the tip of the micropipette. Drying was done under room conditions. Subsequently, the detector was packed using two foils of $10-\mu \mathrm{m}$-thick aluminum overlaid, in order to avoid exposure to light. Aluminum was selected for its small absorption cross section to neutrons and low Fermi potential.

\section{Detection efficiency to cold/ultracold neutrons}

The detector was demonstrated using cold neutrons with a wavelength of $0.2-1.0 \mathrm{~nm}$ at the Low Divergence Beam 

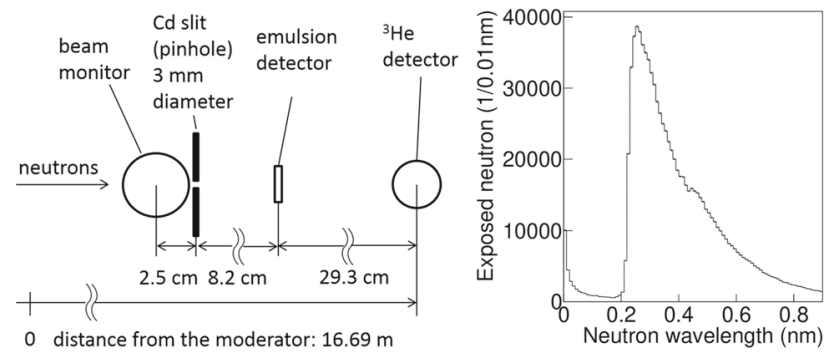

Fig. 2 Left: a schematic view of the experimental setup. Right: the wavelength distribution taken by the ${ }^{3} \mathrm{He}$ detector

Branch of the BL05 $[12,13]$. The experimental setup and the wavelength distribution of the neutrons which was acquired from measurements are shown in Fig. 2. A beam monitor detector (MNH10/4.2F, a ${ }^{3} \mathrm{He}$ proportional counter [14]) was set at the upstream to normalize the beam intensities. A 3mm-diameter pinhole in a cadmium plate was positioned downstream. The machining accuracy of the pinhole was estimated by measuring its radius. It was measured at 10 different positions by using an image scanner with pixel size of $21 \mu \mathrm{m}$. The average radius was $(2.968 \pm 0.019) \mathrm{mm}$ which showed the machining accuracy of $0.64 \%$ to its radius. It affected the estimation of exposure density of cold neutrons to the emulsion detector by $1.3 \%$ and contributed as a part of its systematic uncertainty. The emulsion detector of $2 \mathrm{~cm} \times 2 \mathrm{~cm}$ was set at $8.2 \mathrm{~cm}$ downstream of the pinhole. $\mathrm{A}^{3} \mathrm{He}$ detector (RS-P4-0812-223, a 25.4-mm-diameter 0.97-MPa- ${ }^{3} \mathrm{He}$ proportional counter) was set at its downstream. The intensity and the wavelength of the neutron beam was evaluated by measurement without the emulsion detector. The number of neutrons which passed through the pinhole was evaluated to be $(3.201 \pm 0.004$ (stat) \pm 0.077 (sys) $) \times 10^{6}$ for an exposure time of $22 \mathrm{~min}$, by normalizing the beam monitor counts. The main source of the systematic error was the uncertainty of the normalization which contributes by $2.4 \%$ in relative error.

After the exposure to neutrons, the emulsion detector was developed (photoprocessed) at Nagoya University. Subsequently, tracks from the neutron absorption were observed under an optical microscope with an epi-illumination system, as shown in Fig. 3. Tracks were counted manually in tomographic images taken by a CMOS camera installed in the microscopy system. Only defined sample regions were investigated to minimize the time for counting.

A total of 16 sample regions of $100-\mu \mathrm{m}$ square each in 3-mm diameter corresponding to the pinhole position were scanned for counting and evaluating the detection efficiency. The entire 10- $\mu \mathrm{m}$ thickness of the emulsion layer was investigated. In order to reject small scratch marks on the sputtered layer which mimic the signal tracks, tracks with large angles that were nearly parallel to the sputtered layer were rejected. This reduced the detection efficiency to $(82.5 \pm$ $0.06) \%$ for neutron absorption. This reduction rate was cal-

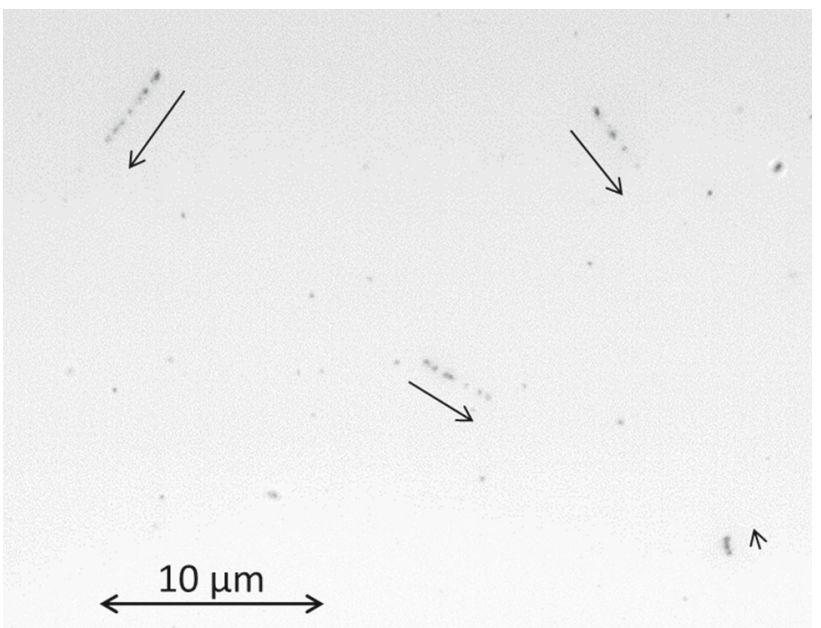

Fig. 3 Tracks from neutron absorption by ${ }^{10} \mathrm{~B}$ were clearly observed under the epi-illumination optical microscope. Arrows are drawn next to tracks. Beginning points of the arrows show starting points of tracks at the surface of the converter layer

culated by taking into consideration the geometry and the intervals of the tomographic images. The uncertainty $0.06 \%$ comes from the depth of field of the microscope, $0.3 \mu \mathrm{m}$. This geometrical cut was the only inefficiency in this measurement. Tracks which were not attached to the sputtered layer or longer than expected ranges were also excluded. A total of 118 absorption events were detected in the 16 regions. 17 tracks were randomly sampled from the detected tracks and their lengths were measured manually in the tomographic images. A length distribution of them is shown in Fig. 4. Measurement errors of lengths were $0.2-0.8 \mu \mathrm{m}$ depending on angles of tracks and existence of Rutherford scattering. The distribution separated into two groups. Average lengths of the tracks in the shorter group and the longer group were $(2.36 \pm 0.19) \mu \mathrm{m}$ and $(4.13 \pm 0.15) \mu \mathrm{m}$, respectively. These lengths should have been shorter than the real ranges depending on the average gap lengths between pairs of adjacent silver grains. Those gap lengths of the shorter and the longer group were measured to be $0.2 \mu \mathrm{m}$ and $0.4 \mu \mathrm{m}$, respectively. By considering those gaps and measurement errors of track lengths, the average lengths of tracks of the two groups were reasonable comparing to ranges of ${ }^{7} \mathrm{Li}$-tracks and $\alpha$-tracks calculated by SRIM-2008.04, $(2.6 \pm 0.4) \mu \mathrm{m}$ and $(5.1 \pm 0.4)$ $\mu \mathrm{m}$. The number of neutrons exposed in this area was (7.25 \pm 0.01 (stat) \pm 0.20 (sys) $) \times 10^{4}$. The main contributions to the systematic error was from uncertainties of the normalization by the beam monitor detector $(2.4 \%)$ and the slit aperture $(1.3 \%)$, in relative errors. The expected detection efficiency was calculated to be $(0.112 \pm 0.001$ (stat) \pm 0.032 (sys))\% using the amount of ${ }^{10} \mathrm{~B}$ in the ${ }^{10} \mathrm{~B}_{4} \mathrm{C}$ layer, which was measured using the cold neutron transmission, the absorption cross section considering the $1 / v$ law, the wavelength distribution in Fig. 2, and the acceptance angle. The main con- 


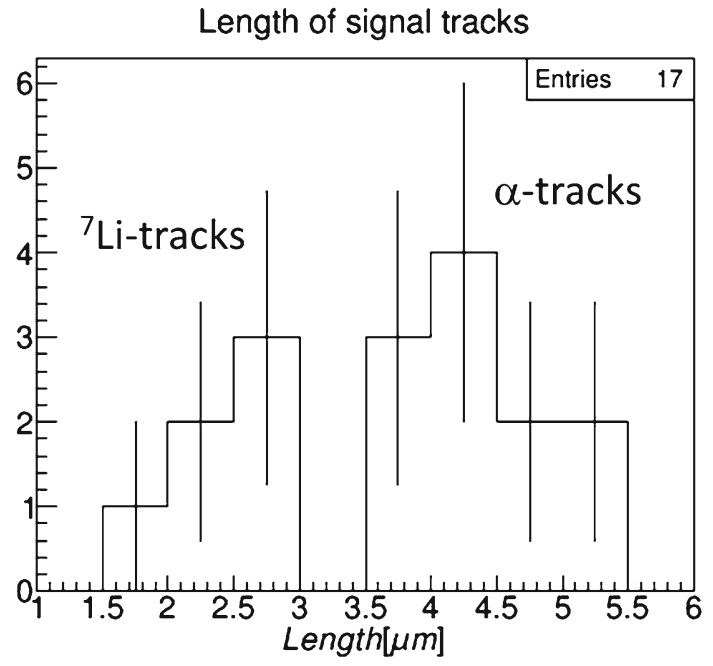

Fig. 4 A length distribution of 17 tracks randomly sampled from detected tracks. It is separated into two groups, the shorter one of ${ }^{7} \mathrm{Li}$ tracks and the longer one of $\alpha$-tracks. An error bar shows a statistical error for each bin

tribution to the systematic error was from the uncertainty of the absorption rate in the ${ }^{10} \mathrm{~B}_{4} \mathrm{C}$ layer as a relative error of $28 \%$. The detection efficiency from this result was $(0.163 \pm$ 0.015 (stat) \pm 0.013 (sys)) $\%$ for the cold neutrons. The main contributions to its systematic error was from uncertainties of the geometrical cut $(7.3 \%)$, normalization by beam monitor detector (2.4\%), and the slit aperture (1.3\%), in relative errors. In those 16 regions, we found 2 proton tracks which were contained in the emulsion layer and not attached to its surface and the sputtered layer. Their length were $(6.0 \pm 0.5)$ $\mu \mathrm{m}$ and $(7.1 \pm 0.6) \mu \mathrm{m}$. Silver grains of those tracks had an average gap of $0.7 \mu \mathrm{m}$. From their lengths and the average grain gap, both of them could be interpreted as from ${ }^{14} \mathrm{~N}(\mathrm{n}, \mathrm{p})$ reactions or proton recoil. From the ${ }^{14} \mathrm{~N}$ content in the emulsion layer, an expected number of ${ }^{14} \mathrm{~N}(\mathrm{n}, \mathrm{p})$ reactions to be occurred in the 16 regions was $1.23 \pm 0.22$. It was consistent with the interpretation. There was no value of expected number of recoiled protons since amount of fast neutrons had not been measured in the beamline we used. Also two $\alpha$-tracks from natural radiations were found. One was penetrating the emulsion layer. The other was entering from the surface of the emulsion layer and stopped in the middle of it.

In order to measure the detection efficiency of the UCN, the detector was exposed to neutrons with a wavelength of 25-100 nm from a Doppler shifter of the BL05 [15], which produces UCNs converted from very cold neutrons (VCNs). The experimental setup is shown in the left schematic of Fig. 5. At the downstream of the UCN port, a square aperture of $1 \times 1 \mathrm{~cm}^{2}$ made on a cadmium plate was positioned. The machining accuracy of the aperture was $1.4 \%$ and $1.2 \%$ to each side of $1 \mathrm{~cm}$. They affected the estimation of the exposure density by $2.6 \%$ and contributed as a part of its
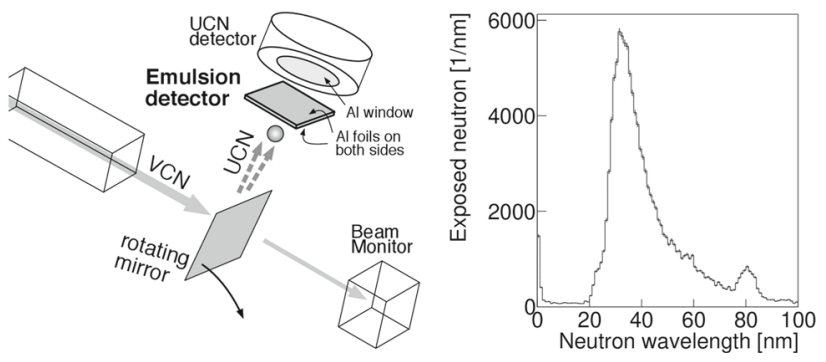

Fig. 5 Left: a schematic view of the experimental set up. Right: the wavelength distribution taken by the UCN detector

systematic uncertainty. The emulsion detector was placed downstream to the aperture using polyimide tape. A UCN detector (DUNia10 [15]) was set at $3.3 \mathrm{~cm}$ downstream of the emulsion detector. The entrance window of this detector was made of $100 \mu \mathrm{m}$-thick aluminum foil. Aluminum was used for its small absorption cross section to neutrons and low Fermi potential. The intensity and the wavelength of the UCN beam was measured using the UCN detector without the emulsion detector in place. The wavelength distribution of the neutrons is shown in the right plot of Fig. 5. The beam monitor used for the cold neutron experiment was set to monitor the flux of the VCNs. This instrument was used to normalize the acquired UCN intensities using the same approach as for the measurement with cold neutrons. The number of UCNs exposed to the emulsion detector was estimated as $(1.45 \pm 0.01$ (stat) \pm 0.10 (sys) $) \times 10^{5}$ for $20.4 \mathrm{~h}$ of exposure. The main contributions to the systematic error was from uncertainties of the difference of detection efficiency by the depth inside the UCN detector (7\%) and transmittance in air $(1.2 \%)$, in relative errors. Attenuation of the UCN flux in the silicon, aluminum, and air were taken into account. That in silicon was obtained from the transmission measurement of cold neutrons. That in aluminum was calculated from cross sections for absorption in Ref. [16] and inelastic scattering in Ref. [17]. That in the air was calculated from the cross section in Ref. [17]. After development of the emulsion, an analysis was performed in the same manner as for the measurement with cold neutrons. In order to reject the scratch marks on the sputtered layer, the same geometrical cut as in cold neutron measurement was applied.

For the calculation of the detection efficiency, the total number of events in the 42 regions of $100-\mu \mathrm{m}$ square each in the $1-\mathrm{cm}$ square at the down stream of the aperture was used. The expected detection efficiency was calculated to be $\left(10.2 \pm 0.1\right.$ (stat) \pm 2.7 (sys))\% using the amount of ${ }^{10} \mathrm{~B}$ in the ${ }^{10} \mathrm{~B}_{4} \mathrm{C}$ layer measured by using the aforementioned transmission of cold neutrons, the absorption cross section considering the $1 / v$ law, the wavelength distribution, and the acceptance angle mentioned above. The main contribution to its systematic error was from the uncertainty of the absorption rate in the ${ }^{10} \mathrm{~B}_{4} \mathrm{C}$ layer as a relative error of $26 \%$. A total of 
63 absorption events were detected in the 42 regions. The total number of neutrons to which this area was exposed was $609 \pm 2$ (stat) \pm 49 (sys). The main contributions to the systematic error was from uncertainties of the difference of detection efficiency by the depth inside the UCN detector $(7 \%)$, slit aperture (2.6\%), and transmittance in air (1.2\%), in relative errors. The detection efficiency from this result was $(10.3 \pm 1.3$ (stat) \pm 1.1 (sys) $) \%$ for the UCNs used in this experiment. The main contribution to its systematic error was from uncertainties of the geometrical cut $(7.3 \%)$, difference of detection efficiency by the depth inside the UCN detector $(7 \%)$, slit aperture $(2.6 \%)$, transmittance in air $(1.2 \%)$, in relative errors. The result was consistent with the expected value of $(10.2 \pm 0.1$ (stat) \pm 2.7 (sys) $) \%$.

In the measurement with cold neutrons, an effective velocity of detected neutrons was calculated as $(995.0 \pm 5.0) \mathrm{m} / \mathrm{s}$ by considering $1 / v$ law. The detection efficiency was converted to that for $2200 \mathrm{~m} / \mathrm{s}$ with $1 / v$ law, which resulted in $(0.074 \pm 0.009) \%$. The errors were combined ones of statistical and systematic. In the measurement with UCNs, the effective velocity was $(9.1 \pm 0.5) \mathrm{m} / \mathrm{s}$. In the same manner as for the measurement with cold neutrons, a detection efficiency for $2,200 \mathrm{~m} / \mathrm{s}$ was calculated to be $(0.043 \pm 0.007) \%$. By taking an weighted average of those two results, we obtained a detection efficiency of $(0.055 \pm 0.006) \%$ for $2200 \mathrm{~m} / \mathrm{s}$ neutrons. It was converted to $(17.4 \pm 1.9) \%$ for $7 \mathrm{~m} / \mathrm{s}$ of UCNs with $1 / v$ law.

\section{Spatial resolution}

The spatial resolution of the absorption points was estimated by fitting the positions of each grain, which was determined from the three-dimensional center of gravity of darkness, in the microscopic image. An example of an $\alpha$-track from an absorption event is shown in Fig. 6. Since the surface of the converter layer reflects light, mirror images of grains are also detected when a focal plane is deeper than the surface. The positions of grains for both real and mirror images were fitted with a pair of lines which met at a point $\mathrm{r}_{0}\left(\mathrm{x}_{0}, \mathrm{y}_{0}\right.$, $\mathrm{z}_{0}$ ), which is the starting point of the track at the surface of the substrate. Position errors at $r_{0}$ were determined from the fitting procedure. They depend on the slope of the tracks. We introduced angles $\theta$ and $\phi$ as shown in Fig. 7. The $\mathrm{x}-\mathrm{y}$ plane shows the surface of the converter layer. To analyze separately the angle-independent contribution and the angledependent one, position errors at $r_{0}$ are described by longitudinal $\left(\delta \mathrm{r}_{\mathrm{L}}\right)$ and transverse $\left(\delta \mathrm{r}_{\mathrm{T}}\right)$ values. $\delta \mathrm{r}_{\mathrm{L}}$ is parallel to the projection of the track to $\mathrm{x}-\mathrm{y}$ plane. The transverse parameter $\delta \mathrm{r}_{\mathrm{T}}$ is perpendicular to it and on the plane. $\delta \mathrm{r}_{\mathrm{L}}$ and $\delta \mathrm{r}_{\mathrm{T}}$ from position data of $7 \alpha$-tracks is shown in Fig. 7. Well fit curves in the figure are dependences of $\delta \mathrm{r}_{\mathrm{L}}$ and $\delta \mathrm{r}_{\mathrm{T}}$ to $\tan \theta$, which are described in equations (3) and (4). In the equations, $a$ is a

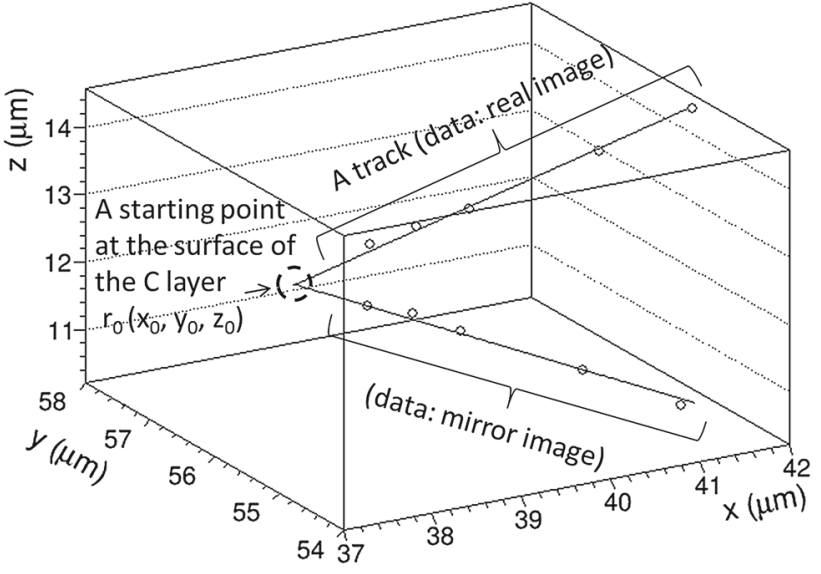

Fig. 6 An example of 3-dimensional position data of grains forming an $\alpha$-track and its mirror image

standard deviation of the residual errors between grain positions and a fitting line in the $\mathrm{x}$ and $\mathrm{y}$ directions. The variable $b$ in (3) is that of the $z$ direction.

$\delta \mathrm{r}_{\mathrm{L}}=a \sqrt{1+(b / a)^{2} \tan ^{2} \theta}$

$\delta \mathrm{r}_{\mathrm{T}}=a$

$\delta \mathrm{r}_{\mathrm{L}}$ can be reduced by selecting tracks with smaller $\tan \theta$. When $\tan \theta$ is restricted, the number of tracks in the acceptance decreases as (5).

$$
1-\frac{1}{\sqrt{1+0.36 \tan ^{2} \theta}}
$$

The factor $0.36 \mathrm{in}$ (5) originates from the shrinkage rate of the emulsion layer during the development. The spatial resolution of the absorption points in the ${ }^{10} \mathrm{~B}_{4} \mathrm{C}$ layer was estimated by considering the extrapolation of tracks from the surface of the converter layer to the middle of the ${ }^{10} \mathrm{~B}_{4} \mathrm{C}$ layer. That of the transverse direction was $11 \mathrm{~nm}$, independent of $\tan \theta$. That of longitudinal direction was $11 \mathrm{~nm}-1.0 \mu \mathrm{m}$ for all slopes of tracks. A higher resolution can be realized with a strict acceptance on $\tan \theta$. For example, when $\tan \theta \leq 1.9$ was set, which excluded all but $34 \%$ of the tracks, a resolution of 11-99 nm was obtained.

\section{Conclusions}

We developed a high spatial resolution neutron detector which was fabricated by coating a converter layer with a fine-grained nuclear emulsion. The converter layer consisted of ${ }^{10} \mathrm{~B}_{4} \mathrm{C}, \mathrm{NiC}$, and $\mathrm{C}$ layers with nominal thickness values of $50 \mathrm{~nm}, 60 \mathrm{~nm}$, and $30 \mathrm{~nm}$, respectively. The detection efficiencies of cold neutrons and UCNs were measured at J-PARC. The results were $(0.163 \pm 0.015$ (stat) \pm 0.013 (sys) $) \%$ and $(10.3 \pm 1.3$ (stat) \pm 1.1 (sys) $) \%$ for the cold 

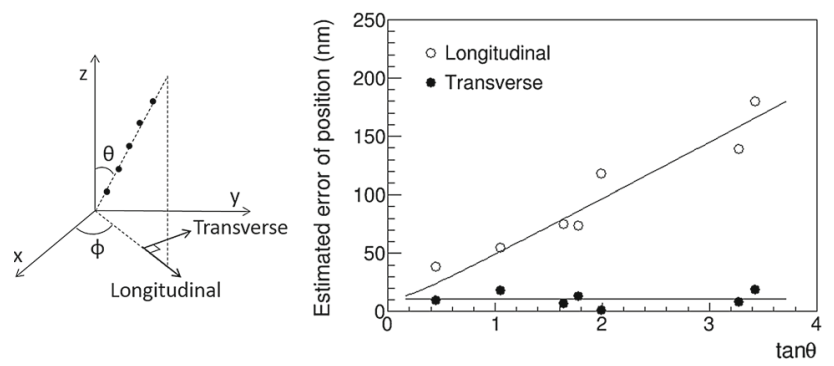

Fig. 7 Left: definition of angles $\theta$ and $\phi$ of a track and directions of longitudinal and transverse errors. The $\mathrm{x}-\mathrm{y}$ plane shows the surface of the converter layer. Right: position errors of detected $\alpha$-tracks in longitudinal and transverse directions in $\mathrm{nm}$ and their dependences on track slopes $(\tan \theta)$

$(\sim 1000 \mathrm{~m} / \mathrm{s})$ and the UCNs $(\sim 10 \mathrm{~m} / \mathrm{s})$, respectively. These values are consistent with the expected values of $(0.112 \pm$ 0.001 (stat) \pm 0.032 (sys)) $\%$ and $(10.2 \pm 0.1$ (stat) \pm 2.7 (sys))\%, respectively. We estimated the spatial resolutions of the absorption points in the ${ }^{10} \mathrm{~B}_{4} \mathrm{C}$ layer using the position data of grains of tracks which resulted from absorptions. The resolution for the transverse direction was $11 \mathrm{~nm}$ and the value obtained for the longitudinal direction depended on the track angle against the surface. Thus, there was a trade-off between resolution and efficiency. When an acceptance angle of $\tan \theta \leq 1.9$ was set, the resolution was $11-99 \mathrm{~nm}$ with $34 \%$ of the statistics. This study is the first to successfully apply nuclear emulsion to the detection of UCNs. The detection efficiency can be increased by optimizing the thickness of the converter layer. An automatic reading out algorithm for scanning larger areas is under development. This type of extremely-high spatial resolution detector can facilitate the pursuit of various experiments involving neutron quantum effects such as the search for short distance forces and the electric charge of neutrons.

Acknowledgements We thank T. Naka, T. Asada, and S. Furuya for providing us fine-grained nuclear emulsion gels, and for their advice regarding their use. We are also grateful to A. Young, T. Ito, and C. Morris for performing test exposure experiments at Los Alamos National Laboratory and fruitful discussions and suggestions on the structure of our detector. Test exposures for previous types of this detector were done at Kyoto University Accelerator-driven Neutron Source with the assistance of M. Hirose and T. Nagae. This work was supported by a JSPS KAKENHI Grant number JP26800132. The experiment was approved by the Neutron Science Proposal Review Committee of J-PARC/MLF (Proposal nos. 2014B0270, 2015A0242, and 2016A0213) and the Neutron Scattering Program Advisory Committee of IMSS, KEK (Proposal no. 2014S03).

Open Access This article is distributed under the terms of the Creative Commons Attribution 4.0 International License (http://creativecomm ons.org/licenses/by/4.0/), which permits unrestricted use, distribution, and reproduction in any medium, provided you give appropriate credit to the original author(s) and the source, provide a link to the Creative Commons license, and indicate if changes were made.

Funded by SCOAP ${ }^{3}$.

\section{References}

1. T. Naka, T. Asada, T. Katsuragawa, M. Yoshimoto, K. Hakamata, K. Kuwabara, M. Nakamura, O. Sato, T. Nakano, Y. Tawara, G. DeLellis, C. Sirignano, N. D'Ambrossio, Fine grained nuclear emulsion for higher resolution tracking detector. Nucl. Instrum. Methods Phys. Res. Sect. A 718, 519-521 (2013)

2. T. Asada, T. Naka, K.I. Kuwabara, M. Yoshimoto, The development of a super-fine-grained nuclear emulsion. Prog. Theor. Exp. Phys. 2017(6), 063H01 (2017)

3. A. Alexandrov, T. Asada, A. Buonaura, L. Consiglio, N. DAmbrosio, G. De Lellis, A. Di Crescenzo, N. Di Marco, M.L. Di Vacri, S. Furuya, G. Galati, V. Gentile a, T. Katsuragawa, M. Laubenstein, A. Lauria, P.F. Loverre, S. Machii, P. Monacelli, M.C. Montesi, T. Naka, F. Pupilli, G. Rosa, O. Sato, P. Strolin, V. Tioukov, A. Umemoto, M. Yoshimoto, Intrinsic neutron background of nuclear emulsions for directional Dark Matter searches. Astropart. Phys. 80, 1621 (2016)

4. V.V. Nesvizhevsky, H. Börner, A.M. Gagarski, G.A. Petrov, A.K. Petukhov, H. Abele, S. Bäßler, T. Stöferle, S.M. Soloviev, Search for quantum states of the neutron in a gravitational field: gravitational levels. Nucl. Instrum. Methods Phys. Res. A 440, 754-759 (2000)

5. Tobias Jenke, Gunther Cronenberg, Hanno Filter, Peter Geltenbort, Martin Klein, Thorsten Lauer, Kevin Mitsch, Heiko Saul, Dominik Seiler, David Stadler, Martin Thalhammer, Hartmut Abele, Ultracold neutron detectors based on ${ }^{10} \mathrm{~B}$ converters used in the qbounce experiments. Nucl. Instrum. Methods Phys. Res. A Accel. Spectrom. Detect. Assoc. Equip. 732, 1-8 (2013)

6. Valery V. Nesvizhevsky, Hans G. Börner, Alexander K. Petukhov, Hartmut Abele, Stefan Baeßler, Frank J. Rueß, Thilo Stöferle, Alexander Westphal, Alexei M. Gagarski, Guennady A. Petrov, Alexander V. Strelkov, Quantum states of neutrons in the earth's gravitational field. Nature 415, 297-299 (2002)

7. H. Abele, T. Jenke, D. Stadler, P. Geltenbort, Qubounce: the dynamics of ultra-cold neutrons falling in the gravity potential of the earth. Nucl. Phys. A 827, 593c-595c (2009)

8. G. Ichikawa, S. Komamiya, Y. Kamiya, Y. Minami, M. Tani, P. Geltenbort, K. Yamamura, M. Nagano, T. Sanuki, S. Kawasaki, M. Hino, M. Kitaguchi, Observation of the spatial distribution of gravitationally bound quantum states of ultracold neutrons and its derivation using the wigner function. Phys. Rev. Lett. 112, 071101 (2014)

9. J. Baumann, R. Gähler, J. Kalus, W. Mampe, Experimental limit for the charge of the free neutron. Phys. Rev. D 37(11), 3107 (1988)

10. C. Siemensen, C. Düsing, P. Geltenbort, C. Giebel, T. Reich, C. Plonka, Search for an electric charge of the neutron. Phys. Rev. D 97, 052004 (2018)

11. Masahiro Hino, Tatsuro Oda, Masaaki Kitaguchi, Norifumi L. Yamada, Seiji Tasaki, Yuji Kawabata, The ion beam sputtering facility at KURRI: Coatings for advanced neutron optical devices. Nucl. Instrum. Methods Phys. Res. A 797, 265-270 (2015)

12. Kenji Mishima, Takashi Ino, Kenji Sakai, Takenao Shinohara, Katsuya Hirota, Kazuaki Ikeda, Hiromi Sato, Yoshie Otake, Hitoshi Ohmori, Suguru Muto, Norio Higashi, Takahiro Morishima, Masaaki Kitaguch, Masahiro Hino, Haruhiko Funahashi, Tatsushi Shima, Jun ichi Suzuki, Koji Niita, Kaoru Taketani, Yoshichika Seki, Hirohiko M. Shimizu, Design of neutron beamline for fundamental physics at J-PARC BL05. Nucl. Instrum. Methods Phys. Res. A 600, 342-345 (2009)

13. Kenji Nakajima et al., Materials and life science experimental facility (MLF) at the Japan proton accelerator research complex II: neutron scattering instruments. Quantum Beam Sci. 1(3), 9-1030009 (2017) 
14. T. Ino, H. Otono, K. Mishima, T. Yamada, Precision neutron flux measurement with a neutron beam monitor. J. Phys. Conf. Ser. 528, 012039 (2014)

15. S. Imajo, K. Mishima, M. Kitaguchi, Y. Iwashita, N.L. Yamada, M. Hino, T. Oda, T. Ino, H.M. Shimizu, S. Yamashita, R. Katayama, Pulsed ultra-cold neutron production using a Doppler shifter at JPARC. Prog. Theor. Exp. Phys. 2016(1), $013 C 02$ (2016)
16. V.F. Sears, Neutron scattering lengths and cross sections. Neutron News 3(3), 26-37 (1992)

17. Albert Steyerl, Herbert Vonach, Total cross-sections of various homogeneous substances for ultracold neutrons. Z. Physik 250, $166-178$ (1972) 